\title{
Reaction crystallization of struvite in a continuous DTM type crystallizer with a compressed air driven jet pump
}

\author{
Andrzej Matynia ${ }^{1 *}$, Agata Mazieńczuk ${ }^{1}$, Krzysztof Piotrowski ${ }^{2}$, Bogusława Wierzbowska ${ }^{1}$, \\ Nina Hutnik ${ }^{1}$, Anna Kozik ${ }^{1}$ \\ ${ }^{1}$ Wroctaw University of Technology, Faculty of Chemistry, Wybrzeże Wyspiańskiego 27, 50-370 Wroctaw, Poland \\ ${ }^{2}$ Silesian University of Technology, Department of Chemical \& Process Engineering, ks. M. Strzody 7, 44-101 Gliwice, \\ Poland \\ "Corresponding author: e-mail: andrzej.matynia@pwr.wroc.pl
}

\begin{abstract}
Experimental tests covering the production of struvite $\mathrm{MgNH}_{4} \mathrm{PO}_{4} \cdot 6 \mathrm{H}_{2} \mathrm{O}$ from water solutions containing 1.0 mass $\%$ of phosphate $(\mathrm{V})$ ions using magnesium and ammonium ions in stoichiometric proportions were carried out in a crystallizer of $1.2 \mathrm{dm}^{3}$ working volume. The process temperature was $298 \mathrm{~K}$. Struvite crystals of mean size $L_{\mathrm{m}}$ from ca. 14 to ca. $38 \mu \mathrm{m}$ were produced depending on the process environment's $\mathrm{pH}$ (9-11) and the mean residence time of suspension in a crystallizer, $\tau(900-3600 \mathrm{~s})$. In such defined process conditions the linear growth rate of struvite crystals changed from $1.45 \cdot 10^{-8} \mathrm{~m} / \mathrm{s}(\mathrm{pH} 9, \tau 900 \mathrm{~s})$ to $2.06 \cdot 10^{-9}$ $\mathrm{m} / \mathrm{s}(\mathrm{pH} 11, \tau 3600 \mathrm{~s})$ while the nucleation rate from $5.1 \cdot 10^{7}$ to $3.2 \cdot 10^{9} 1 /\left(\mathrm{sm}^{3}\right)$. Crystal product of the most advantageous granular characteristics was produced at $\mathrm{pH} 9$ and the mean residence time $3600 \mathrm{~s}$. Within this product population the largest sizes reached above $200 \mu \mathrm{m}$ while the number of crystals smaller than $3 \mathrm{~mm}$ was kept below $6 \%$.
\end{abstract}

Keywords: continuous DTM crystallizer, jet pump, compressed air, reaction crystallization, phosphates(V) removal, struvite, crystal size distribution, SIG kinetic model.

\section{INTRODUCTION}

In most of the operated on industrial scale DTM (Draft Tube Magma) type crystallizers internal circulation of suspension is the effect of mixer or pump action ${ }^{1}$. It can be also realized by the properly designed jet pumps ${ }^{2-4}$. The jet pump can be fed with mother solution collected from the crystallizer's overflow ${ }^{5-7}$ or with compressed air $^{8}$. Compressed air (or another gaseous agent if necessary from the technological requirements) provided into the feeding nozzle of a jet pump becomes thus a working agent, inducing permanent internal circulation and mixing of the suspension in the working volume of the apparatus. After such rearrangement of the original liquid jet pump construction, the crystallizer's overflow and the external circulating pump can be eliminated from the plant. In general, the jet pump apparatus construction makes the removal of the suspension of representative in respect to the bulk magma product crystal size distribution, possible. It can be thus roughly assumed that the DTM type crystallizer with the jet pump driven by compressed air stream fulfills theoretical assumptions defining the MSMPR (Mixed Suspension Mixed Product Removal) crystallizer model ${ }^{1-3}$. It thus becomes possible to estimate the values of fundamental kinetic parameters of the process based on the product crystal size distributions (CSDs) ${ }^{9}$.

Chemical removal of phosphate(V) ions from waste solutions relies upon chemical binding of these species in a form of their sparingly soluble magnesium and ammonium salt $\mathrm{MgNH}_{4} \mathrm{PO}_{4} \cdot 6 \mathrm{H}_{2} \mathrm{O}$, struvite, MAP (solubility product $\left.\mathrm{p} K_{\mathrm{sp}}=9.4-13.26^{10}\right)$. Struvite precipitates at ambient temperature from the supersaturated solutions of magnesium phosphate $(\mathrm{V})$ in the presence of ammonium ions within $\mathrm{pH} 7-11^{11}$. The process course and the results are mainly dependent on: $\mathrm{pH}$, working supersaturation in mother solution, temperature, the presence of the dissolved and solid impurities, the mixing intensity and crystallizer design ${ }^{7,8,10-13}$. In modern phosphorus recycling processes struvite production results from the contacting of magnesium and ammonium ions (e.g. water solution of magnesium chloride and some ammonium salt) with the initially purified municipal, industrial or agricultural liquid wastes (e.g. liquid manure) containing phosphate $(\mathrm{V})$ ions in strictly specified and controlled chemical and hydrodynamical conditions resulting from a properly designed crystallizer construction ${ }^{13-15}$. The effectiveness of struvite reaction crystallization process is thus determined by the crystallizer type and mode of operation, constructional solution of its interior, spatial distribution of reagent inlet ports, as well as the proper selection of its continuous work parameters ${ }^{10,13}$. Besides the essential for the environment protection effect of liquid wastes purification, thus effective prevention of, among others, disadvantageous processes of eutrophication in natural inland water regions, separated crystal product is regarded to be significant secondary source of scarce phosphorus. The recovered struvite can be a subject of further processing or direct usage in agriculture as in wide demand mineral fertilizer ${ }^{\mathbf{1 6}}$.

The research results concerning the production of struvite from the water solutions containing phosphate $(\mathrm{V})$ ions in DTM MSMPR type crystallizer with jet pump driven by compressed air stream are presented and discussed. From the product CSDs, rearranged into population density distributions, fundamental kinetic parameters of the reaction crystallization process were estimated. Calculations were based on the most simplified kinetic model of classical mass crystallization process in MSMPR crystallizer - SIG (Size Independent Growth) model. This approach assumes that in the defined process conditions the linear growth rate of all crystals is identical, thus independent of their individual sizes and surface morphology. 


\section{EXPERIMENTAL AND METHODS}

The experimental plant scheme is presented in Fig. 1. The laboratory scale DTM MSMPR crystallizer with a jet pump driven by compressed air stream was a cylindrical tank made with Plexiglas of $90 \mathrm{~mm}$ diameter and $330 \mathrm{~mm}$ of total height. Crystallizer's working volume was $1.2 \mathrm{dm}^{3}$. The feeding nozzle of the jet pump was fixed in the apparatus bottom resulting in the arrangement of the upward suspension movement in a mixing chamber, followed by its turning back downward in the upper draft tube profile of the internal diameter $60 \mathrm{~mm}$. The geometrical proportions within the crystallizer interior and jet pump hydraulic system, its detailed technical specification, experimental and product analysis procedures are presented in detail in other authors' work ${ }^{\mathbf{8}}$. The apparatus was continuously provided with a feed of the concentration 1.0 mass \% of phosphate(V) ions and - in stoichiometric proportions - with other ions of concentrations: $\left[\mathrm{Mg}^{2+}\right]=0.256$ and $\left[\mathrm{NH}_{4}{ }^{+}\right]=0.190$ mass \%, respectively. The initially blended liquid reagents were introduced into the process environment as ammonium dihydrogenphosphate(V) $\mathrm{NH}_{4} \mathrm{H}_{2} \mathrm{PO}_{4}$, magnesium chloride $\mathrm{MgCl}_{2} \cdot 6 \mathrm{H}_{2} \mathrm{O}$ (both p.a., POCh, Gliwice, Poland) and deionized water (Barnstead - NANOpure DIamond). The process temperature was $298 \pm 0.2 \mathrm{~K}$, while the environment's $\mathrm{pH}$ was adjusted with water solution of $\mathrm{NaOH}(5$ mass \%) to 9,10 and $11( \pm 0.1)$, depending on the current test requirements. The mean residence time $\tau$ of the suspension in a crystallizer working volume was changed from 900 to $3600 \mathrm{~s}( \pm 20 \mathrm{~s})$. The process temperature, $\mathrm{pH}$ and inlet / outlet stream flows (feed solution / product crystal magma) were strictly controlled with PC computer driven by IKA Labworldsoft software. The inlet ports of the reagents and alkaline agent solution, compressed air stream, as well as product suspension outlet are schematically marked in Fig. 1. The jet pump's feeding nozzle was provided with a minimal volumetric stream of compressed air $\left(q_{\mathrm{ve}}=0.37 \mathrm{dm}^{3} / \mathrm{s}\right)$, necessary only to keep all solid particles in a permanent movement. The intensity of internal circulation and induced mixing was thus also minimal. This way the lower boundary values of hydrodynamic parameters of struvite reaction crystallization in such defined process conditions were determined experimentally.

A continuous reaction crystallization process of struvite synthesis followed by its separation from mother solution in a crystalline form ran through the time of $5 \tau$ (starting from the moment of stabilization of the assumed flow process parameter values). After this time the whole crystallizer content was transferred into the vacuum filter for mother liquor drainage. The crystals were not washed but weighed before and after drying. Also the mass and volume of mother solution were determined. This way the solid phase concentration in a product crystal suspension $\left(M_{\mathrm{T}}\right)$ could be calculated. The product crystal size distribution was determined with the solid particle analyzer COULTER LS-230, whereas their habits were evaluated from scanning electron microscope JEOL JSM 5800LV images. The chemical composition of mother solution and solid phase were determined applying, among others, plasma emission spectrometer ICP-AES CPU 7000, spectrometer IR PU9712, spectrophotometer UV-VIS

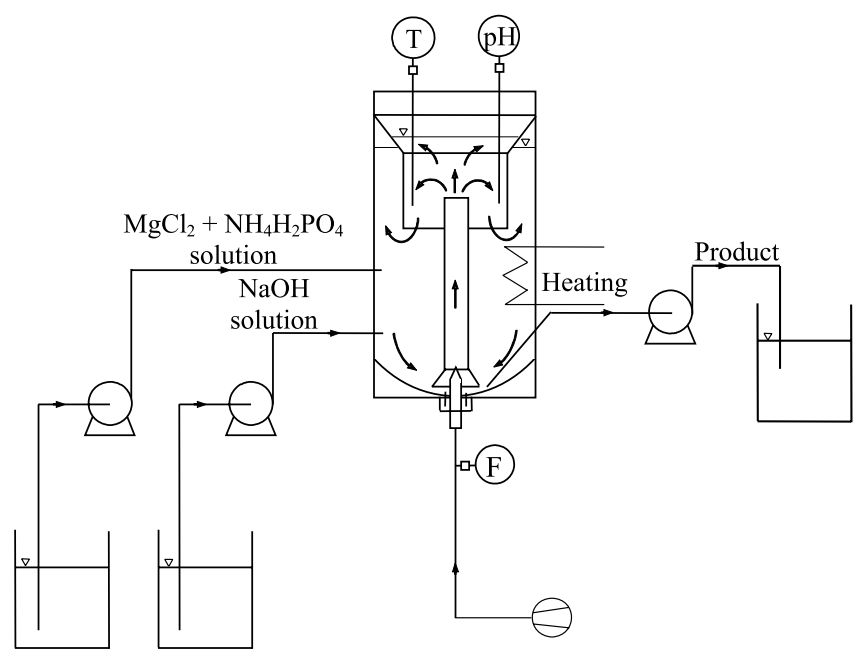

Figure 1. A scheme of laboratory scale plant composed of DTM MSMPR crystallizer with a jet pump driven by compressed air stream and auxiliary equipment

Evolution 300. The accuracy of the measurement data collected in a continuous laboratory plant was estimated to be $10-15 \%$. Thus in Table 1 the mean values of $M_{\mathrm{T}}$ and phosphate $(\mathrm{V})$ ions concentration in a postprocessed mother solution are provided.

The automatically determined with the use of particle analyzer COULTER LS-230 product CSDs were then converted into population density distributions ${ }^{1}$. The individual population density values, ni, were determined based on the original mass $m(L)$ (or volume, $V(L)$ ) size distribution data according to Eq.(1):

$n_{\mathrm{i}}=\frac{m_{\mathrm{i}}}{k_{\mathrm{v}} \rho L_{\mathrm{i}}^{3} \Delta L_{\mathrm{i}} V_{\mathrm{w}}}=\frac{V_{\mathrm{i}}}{k_{\mathrm{v}} L_{\mathrm{i}}^{3} \Delta L_{\mathrm{i}} V_{\mathrm{w}}}$

Elaboration of the experimental data was based on a continuous MSMPR crystallizer - SIG kinetic model9:

$n(L)=n_{0} \exp \left(-\frac{L}{G \tau}\right)$

Eq. (2) enables one to determine the values of fundamental kinetic parameters of a mass (reaction-)crystallization process in a theoretically idealized MSMPR crystallizer configuration - nucleation and growth rates. The plot of $\ln n$ versus $L$ gives a straight line with an intercept at $L=0$ equals to $\ln n_{0}$ and a slope interpreted as $-1 / G \tau$. If the mean residence time of suspension, $\tau$, can be experimentally adjusted with satisfactory accuracy (e.g. computer control system), linear growth rate of crystals, $G$, can be calculated directly.

From the nuclei population density, $\mathrm{n}_{0}$ and linear growth rate, $G$, the value of nucleation rate $B$ can be determined, Eq. (3):

$B=n_{0} G$

\section{RESULTS AND DISCUSSION}

All research results are presented in Table 1. From these data it results that the crystal size of the product decreases with the increase in the process environment's pH (assuming other parameter values constant). The increase in $\mathrm{pH}$ from 9 to 11 resulted that mean crystal size $L_{\mathrm{m}}$ decreased by ca. $47 \%$ for $\tau 900 \mathrm{~s}\left(L_{\mathrm{m}}=27.3 \rightarrow 14.4 \mu \mathrm{m}\right)$, and even above $55 \%$ for $\tau 1800$ and $3600 \mathrm{~s}$. With the $\mathrm{pH}$ parameter 
Table 1. Influence of selected technological input parameters $(\mathrm{pH}, \tau)$ of struvite reaction crystallization process in a continuous DTM MSMPR crystallizer with jet pump driven by compressed air stream on the final product crystal properties

\begin{tabular}{|c|c|c|c|c|c|c|}
\hline \multirow{2}{*}{ No. } & \multicolumn{2}{|c|}{ Technological parameters } & \multicolumn{3}{|c|}{ Product properties } & \multirow{2}{*}{$L_{m}^{17,18}, \mu \mathrm{m}$} \\
\hline & $\mathrm{pH}$ & $\tau, \mathbf{s}$ & $L_{\mathrm{m}}, \mu \mathrm{m}$ & $L_{50}, \mu \mathrm{m}$ & CV, \% & \\
\hline 1 & 9 & 900 & 27.3 & 20.7 & 88.6 & 26.0 \\
\hline 2 & 10 & 900 & 20.1 & 13.4 & 94.3 & 22.5 \\
\hline 3 & 11 & 900 & 14.4 & 9.6 & 103.0 & 21.3 \\
\hline 4 & 9 & 1800 & 34.3 & 26.1 & 87.8 & 42.3 \\
\hline 5 & 10 & 1800 & 22.0 & 17.5 & 89.1 & 26.0 \\
\hline 6 & 11 & 1800 & 14.8 & 10.6 & 94.9 & 20.7 \\
\hline 7 & 9 & 3600 & 38.1 & 28.4 & 88.9 & 41.3 \\
\hline 8 & 10 & 3600 & 22.3 & 16.0 & 86.2 & 24.4 \\
\hline 9 & 11 & 3600 & 16.7 & 12.7 & 97.3 & 20.8 \\
\hline
\end{tabular}

$T=298 \mathrm{~K}$

Concentrations of substrate ions in a feed:

$\left[\mathrm{PO}_{4}{ }^{3-}\right]=1.0$ mass $\%,\left[\mathrm{Mg}^{2+}\right]=0.256$ mass $\%,\left[\mathrm{NH}_{4}{ }^{+}\right]=0.190$ mass $\%$

Average concentration of phosphate $(\mathrm{V})$ ions in a postprocessed mother solution: $0.011 \pm 0.001$ mass \%

Average concentration of struvite crystals in suspension $M_{\mathrm{T}} 24.6 \pm 0.4 \mathrm{~kg} / \mathrm{m}^{3}$

Table 2. Nucleation $(B)$ and linear growth $(G)$ rates of struvite crystals in reaction crystallization process - continuous DTM MSMPR crystallizer with jet pump driven by compressed air stream

\begin{tabular}{|c|c|c|c|c|}
\hline $\begin{array}{c}\text { No. } \\
\text { (see Table 1) }\end{array}$ & Detailed form of $n(L)^{*}$ (Eq. (2)) & $R^{2}$ & $G, \mathrm{~m} / \mathrm{s}$ & $B, 1 /\left(\mathrm{m}^{3} \mathrm{~s}\right)$ \\
\hline 1 & $n=2.798 \cdot 10^{16} \exp \left(-7.666 \cdot 10^{4} L\right)$ & 0.994 & $1.45 \cdot 10^{-8}$ & $4.1 \cdot 10^{8}$ \\
2 & $n=4.121 \cdot 10^{16} \exp \left(-9.155 \cdot 10^{4} L\right)$ & 0.993 & $1.21 \cdot 10^{-8}$ & $5.0 \cdot 10^{8}$ \\
3 & $n=4.178 \cdot 10^{17} \exp \left(-1.445 \cdot 10^{5} L\right)$ & 0.996 & $7.69 \cdot 10^{-9}$ & $3.2 \cdot 10^{9}$ \\
4 & $n=2.015 \cdot 10^{16} \exp \left(-6.677 \cdot 10^{4} L\right)$ & 0.993 & $8.32 \cdot 10^{-9}$ & $1.7 \cdot 10^{8}$ \\
5 & $n=1.871 \cdot 10^{16} \exp \left(-1.040 \cdot 10^{5} L\right)$ & 0.995 & $5.34 \cdot 10^{-9}$ & $1.0 \cdot 10^{8}$ \\
6 & $n=4.475 \cdot 10^{17} \exp \left(-1.471 \cdot 10^{5} L\right)$ & 0.997 & $3.78 \cdot 10^{-9}$ & $1.7 \cdot 10^{9}$ \\
7 & $n=1.048 \cdot 10^{16} \exp \left(-5.750 \cdot 10^{4} L\right)$ & 0.996 & $4.83 \cdot 10^{-9}$ & $5.1 \cdot 10^{7}$ \\
8 & $n=1.787 \cdot 10^{16} \exp \left(-9.424 \cdot 10^{4} L\right)$ & 0.992 & $2.95 \cdot 10^{-9}$ & $5.3 \cdot 10^{7}$ \\
9 & $n=2.634 \cdot 10^{17} \exp \left(-1.347 \cdot 10^{5} L\right)$ & 0.994 & $2.06 \cdot 10^{-9}$ & $5.4 \cdot 10^{8}$ \\
\hline
\end{tabular}

${ }^{*}$ Valid for: $L>40 \mu \mathrm{m}(\mathrm{pH} 9), L>30 \mu \mathrm{m}(\mathrm{pH} 10)$ and $L>20 \mu \mathrm{m}(\mathrm{pH} 11) ; k_{\mathrm{v}}=1$

increase the nuclei population density of struvite increases (see Table 2), resulting in the shifting of the mean product crystal size towards lower values.

Two-time prolongation of the mean residence time of suspension in a crystallizer (from 900 to $1800 \mathrm{~s}$ ) at $\mathrm{pH}$ 9 resulted in an increase of the mean product crystal size by ca. $25 \%: L_{\mathrm{m}}=27.3 \rightarrow 34.3 \mu \mathrm{m}$. This is the effect of working supersaturation decrease, inducing thus appropriate decreases in the nonlinearly correlated nucleation rate $B$ and crystal linear growth rate $G$ values (see Table 2). However, elongation of the mean residence time of crystal suspension compensates this disadvantageous decrement in a $G$ value, arranging thus longer and more stable size enlargement process conditions. It must be noted that such change is also responsible for the increase in attrition and breakage intensities, observed especially within larger crystal fractions and for longer mean residence times (e.g. for $\tau 3600 \mathrm{~s}$ at pH 9: $L_{\mathrm{m}}=38.1 \mu \mathrm{m}$, thus increase in size only by about $4 \%$ compared to $L_{\mathrm{m}}=34.3 \mu \mathrm{m}$ for $\mathrm{t} 1800 \mathrm{~s}$ ). At higher $\mathrm{pH}$ levels the less pronounced increments in the particle size were observed with systematic elongations of the mean residence time of the suspension in a crystallizer (see Table 1). Elongation of this time from 900 to $1800 \mathrm{~s}$ produced at $\mathrm{pH} 10$ increase in $L_{\mathrm{m}}$ by less than $10 \%$ while at $\mathrm{pH} 11$ - only by ca. $3 \%$. The disadvantageous influence of $\mathrm{pH}$ on the linear growth rate $G$ of struvite crystals is more significant (see Table 2).

In Table 1 there are also presented the resulting parameters of crystalline struvite produced in the same reaction crystallization process conditions and in the identical crystallizer configuration, however fed with the solutions of 5-time lower concentration of phosphate(V) ions ${ }^{17,18}$. After a detailed comparison of both data series it results that from the less concentrated solutions the products of larger mean crystal sizes (by 20\% in average) were produced. It can be explained, among others, by the lower supersaturation in a crystallizer (thus lower primary nucleation rate), as well as by the lower intensity of crystals attrition (secondary nucleation) resulting from their 5time lower concentration in the suspension $\left(M_{\mathrm{T}}=4.80\right.$ and $24.6 \mathrm{~kg} / \mathrm{m}^{3}$ for the concentrations of phosphate $(\mathrm{V})$ ions in a feed 0.20 and 1.0 mass \%, respectively). In both cases the average concentration of phosphate $(V)$ ions in a postprocessed mother liquor after reaction crystallization of struvite was low and practically identical: 0.010 0.013 mass $\%^{17,18}$ (Table 1 ).

A simultaneous influence of $\mathrm{pH}$ within the 9-11 range and the mean residence time of suspension in a crystallizer $900<\tau<3600 \mathrm{~s}$ on mean size of struvite crystals, $L_{\mathrm{m}}$ (in $\mu \mathrm{m})$ can be described using empirical correlation, Eq. (4): $L_{\mathrm{m}}=5.17 \times 10^{4} \mathrm{pH}^{-3.83} \tau^{0.141}$

With the correlation coefficient $R^{2}=0.978$ and mean relative error $\pm 4.8 \%$. Graphical projection of this empirical dependency was presented in Fig. 2.

Struvite product crystals of very diversified sizes were removed from the crystallizer. From the data presented in Table 1 it is visible that the coefficient of crystal size variation $\mathrm{CV}$ demonstrated relatively large values, from 


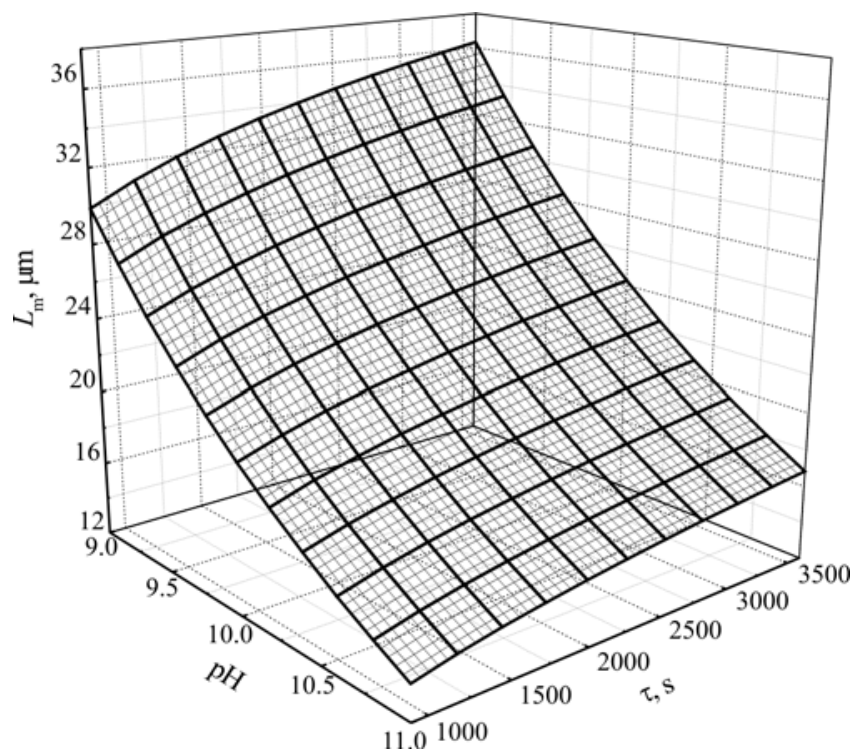

Figure 2. Simultaneous influence of $\mathrm{pH}$ and the mean residence time $\tau$ in a continuous DTM MSMPR crystallizer with a jet pump driven by compressed air stream on the mean crystal size $L_{\mathrm{m}}$ of struvite, Eq. (4). The process temperature $298 \mathrm{~K}$, concentration of phosphate( $(\mathrm{V})$ ions in a feed: 1.0 mass $\%$

within the $86.2-103 \%$ range. However, none unequivocal correlation between the homogeneity in the product crystals population $\mathrm{CV}$ and the corresponding process parameters $(\mathrm{pH}, \tau)$ was statistically identified. It may be supposed, that no evidence of clear tendency within the experimental data set of $\mathrm{CV}=\mathrm{f}(\mathrm{pH}, \tau)$ form is the net effect of complex interactions between the $\mathrm{pH}$ and the mean residence time of the suspension, as well as contribution of attrition and breakage within the crystal phase to the self-establishment of a working supersaturation level in a crystallizer. It should be also noticed that oscillations of crystallizer working parameters $(T \pm 0.2 \mathrm{~K}, \mathrm{pH} \pm 0.1$, $\tau \pm 20 \mathrm{~s}$ ), unavoidable in experimental practice in spite of computer control of a laboratory plant performance, as well as analytical procedure errors, especially concerning the evaluation of crystal size distributions of the dry product, also contribute to the precision of kinetic data determination.

In Fig. 3 there are presented some exemplary volumetric (mass) size distributions of the product crystals corresponding to $\mathrm{pH} 9,10$ and 11 for $\tau 900 \mathrm{~s}$ and to $\mathrm{pH} 9$ for $\tau 3600 \mathrm{~s}$, respectively. With the increase in the process environment's $\mathrm{pH}$ - assuming constant $\tau$ - dominant crystal size $\left(L_{\mathrm{d}}\right.$, corresponding to maximum in a differential distribution course) shifted towards lower crystal sizes: $L_{\mathrm{d}}=24.2 \mu \mathrm{m}\left(\mathrm{pH} \mathrm{9)}, L_{\mathrm{d}}=20.7 \mu \mathrm{m}(\mathrm{pH} \mathrm{10})\right.$ and $L_{\mathrm{d}}=$ $13.5 \mu \mathrm{m}(\mathrm{pH} 11)$. The raise of $\mathrm{pH}$ resulted also in the decrease of the number and size of the largest crystals in a product. The largest size of struvite particles produced at pH 9 reached ca. $160 \mu \mathrm{m}$ (Fig. 3a), at pH $10-$ ca. 120 $\mu \mathrm{m}$ (Fig. 3b), while at pH 11 - only ca. $80 \mu \mathrm{m}$ (Fig. 3c). At the same time a fraction of smaller particles enlarged. At $\mathrm{pH} 9$ crystal fraction of the sizes below $3 \mu \mathrm{m}$ was $7.8 \%$, while at $\mathrm{pH} 11$ it increased up to $15.2 \%$, thus the 2-time increase was reported. In effect the mean crystal size $L_{\mathrm{m}}$ decreased considerably from 27.3 to $14.4 \mu \mathrm{m}$ (see Table 1).

Elongation of the mean residence time of crystal suspension in a crystallizer from 900 to $3600 \mathrm{~s}$ (at $\mathrm{pH}$ 9) caused that the dominant size of the product crystals $L_{\mathrm{d}}$ increased from 24.2 to $33.9 \mu \mathrm{m}$, while the largest crystal sizes also demonstrated an advantageous growing tendency - from ca. 160 to $210 \mu \mathrm{m}$ (Fig. 3d). Crystal fraction attributed to the sizes smaller than $3 \mu \mathrm{m}$ decreased (from 7.8 to $5.9 \%$ ) in spite of the fact that with the elongation of the mean residence time crystal attrition and breakage effects within the bulk of circulated suspension increase considerably.

In Fig. 4 there are presented scanning electron microscope images of struvite crystals, whose size distributions are presented in Fig. 3. The smaller sizes of the particles
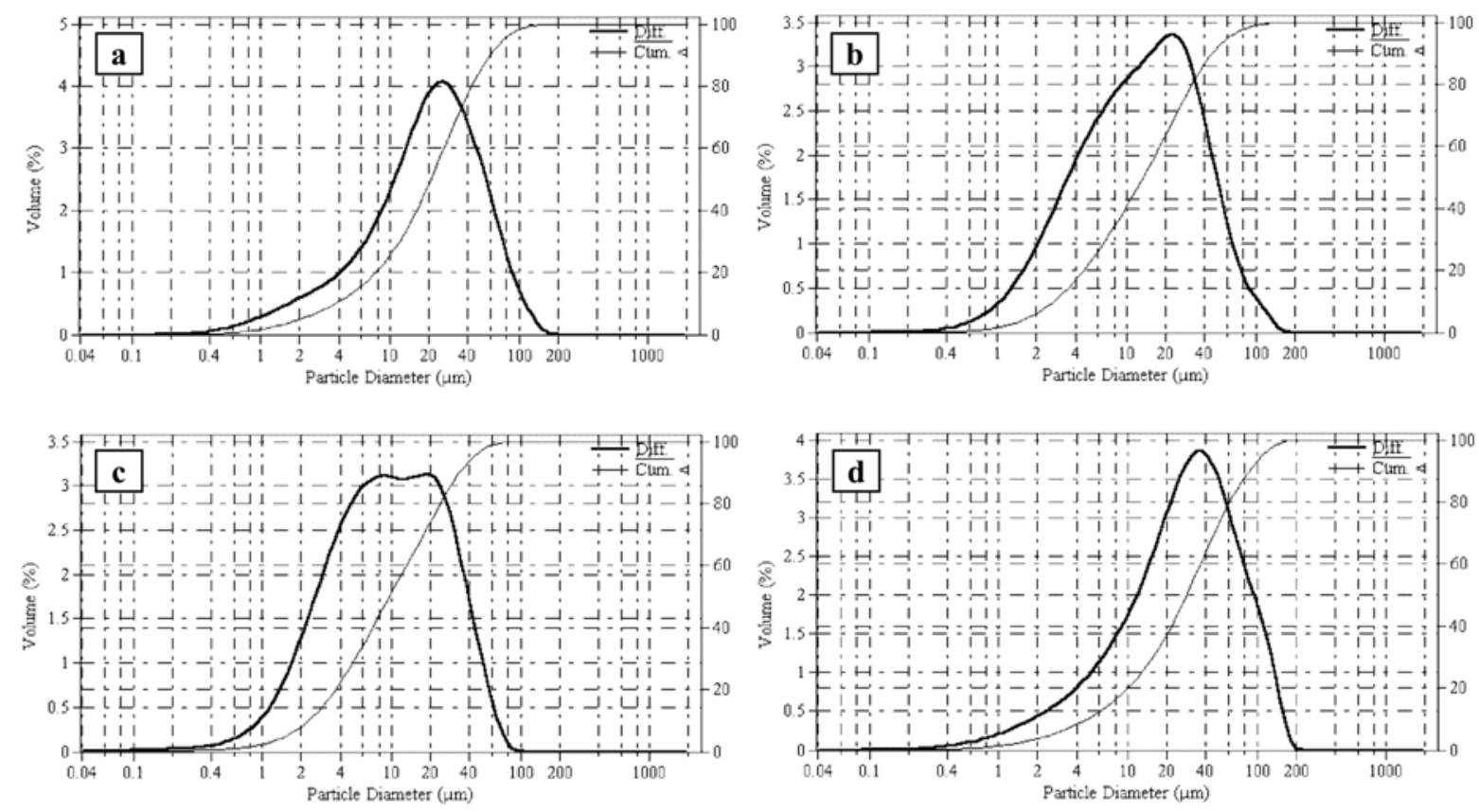

Figure 3. Exemplary differential (left scale) and cumulative (right scale) volumetric (mass) size distributions of struvite crystals produced in a continuous DTM MSMPR crystallizer with a jet pump driven by compressed air stream: a) $\mathrm{pH} 9, \tau 900$ s, b) $\mathrm{pH} 10, \tau 900 \mathrm{~s}, \mathrm{c}) \mathrm{pH} \mathrm{11, \tau} 900 \mathrm{~s}$ and d) $\mathrm{pH} \mathrm{9, \tau} 3600 \mathrm{~s}$ (a, b, c and d correspond to No. 1, 2, 3 and 7 in Tab. 1) 

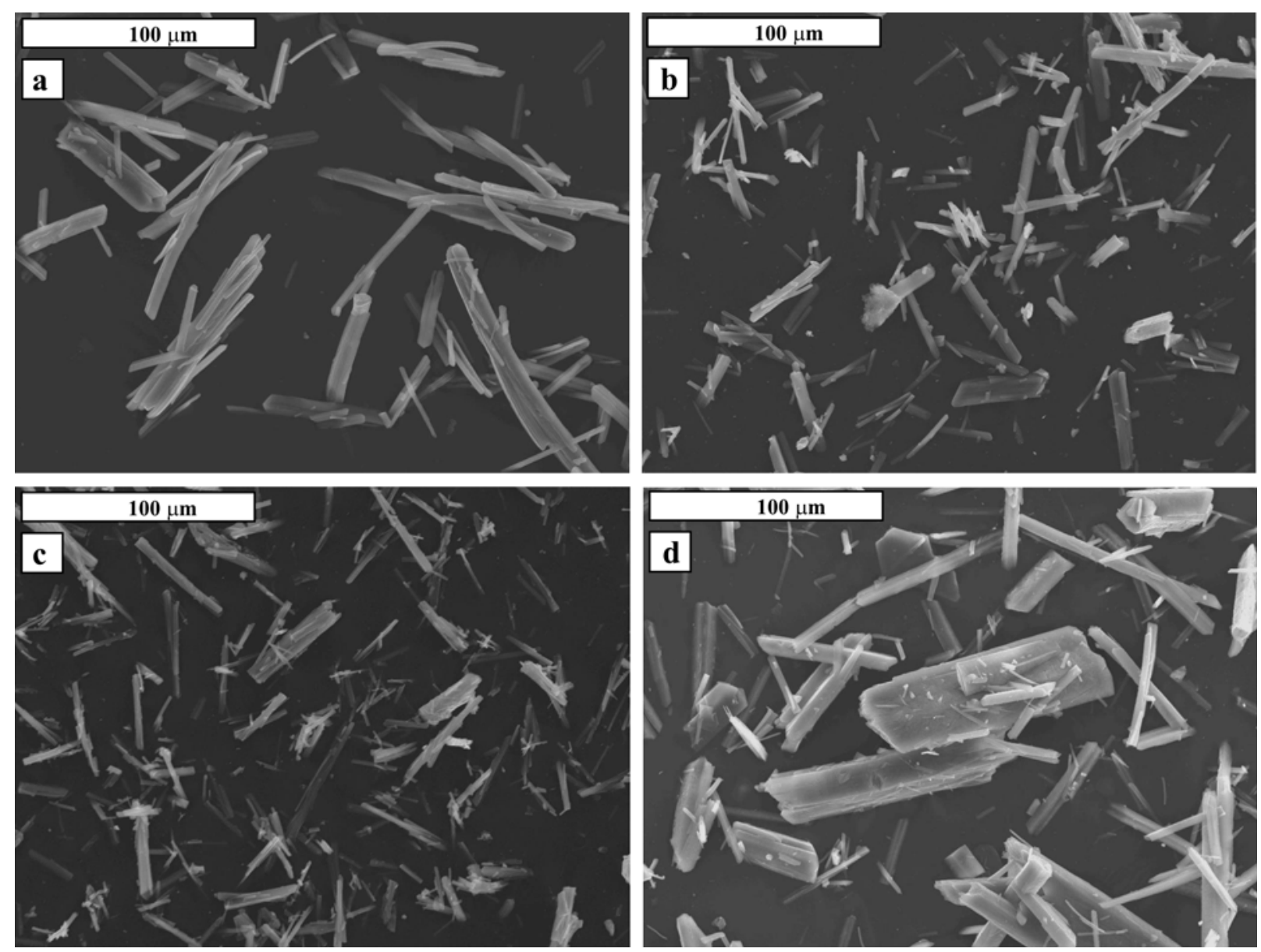

Figure 4. Exemplary scanning electron microscope images of struvite crystals produced in DTM MSMPR crystallizer with jet pump driven by compressed air stream: a) $\left.\left.L_{\mathrm{m}}=27.3 \mu \mathrm{m}(\mathrm{pH} \mathrm{9, \tau} 900 \mathrm{~s}), \mathrm{b}\right) L_{\mathrm{m}}=20.1 \mu \mathrm{m}(\mathrm{pH} 10, \tau 900 \mathrm{~s}), \mathrm{c}\right)$ $L_{\mathrm{m}}=14.4 \mu \mathrm{m}(\mathrm{pH} 11, \tau 900 \mathrm{~s})$, d) $L_{\mathrm{m}}=38.1 \mu \mathrm{m}(\mathrm{pH} 9, \tau 3600 \mathrm{~s})$, magnification $500 \mathrm{x}$, cf. Fig. 3 for size distribution details

corresponding to higher $\mathrm{pH}$ of struvite reaction crystallization environment are clearly observable. From the detailed analysis of crystal population images it also results, that with the increase in the process environment $\mathrm{pH}$ struvite crystals become thinner. Crystal attrition and breakage during their intensive mixing and circulation in the crystallizer can be regarded moderate. Single breaked crystals, surface fragmentation and destruction effects, rounded edges etc. were only observed, however the number of these crystals was not large and it did not dominate the planimetric characteristics of the whole population.

In Fig. 5 there are presented experimental population density distributions of struvite product crystals corresponding to $\mathrm{pH} \mathrm{9,10}$ and 11 . The mean residence time of the suspension in a crystallizer was set constant (900 s). The influence of the mean residence time of the crystals (900, 1800 and $3600 \mathrm{~s}$ ) in a crystallizer working volume - at constant pH 9 - on their population density distributions is, in turn, demonstrated in Fig. 6. The population density values $n(L)$ were calculated with Eq. (1) assuming crystal volumetric shape factor $k_{\mathrm{v}}=1$. From these distribution courses, presented in $\ln n-L$ coordinate system, it results that for struvite particles of size $L>40 \mu \mathrm{m}$ (pH 9), $L>30 \mu \mathrm{m}(\mathrm{pH} 10)$ or $L>20 \mu \mathrm{m}(\mathrm{pH} 11)$ corresponding segments of these dependencies can be with satisfactory precision approximated with the linear function (characteristic feature of SIG kinetic model). Assuming the linearity of $\ln n(L)$ function segment one can cal- culate from Eq. (2) crystal linear growth rate $G$, since from Eq. (3) their nucleation rate, $B$. The detailed forms of population density distributions of struvite product crystals $n(L)$ (Eq. (2)) and calculated on this basis $G$ and $B$ values, corresponding to selected $(\mathrm{pH}, \tau)$ pairs, are presented in Table 2 .

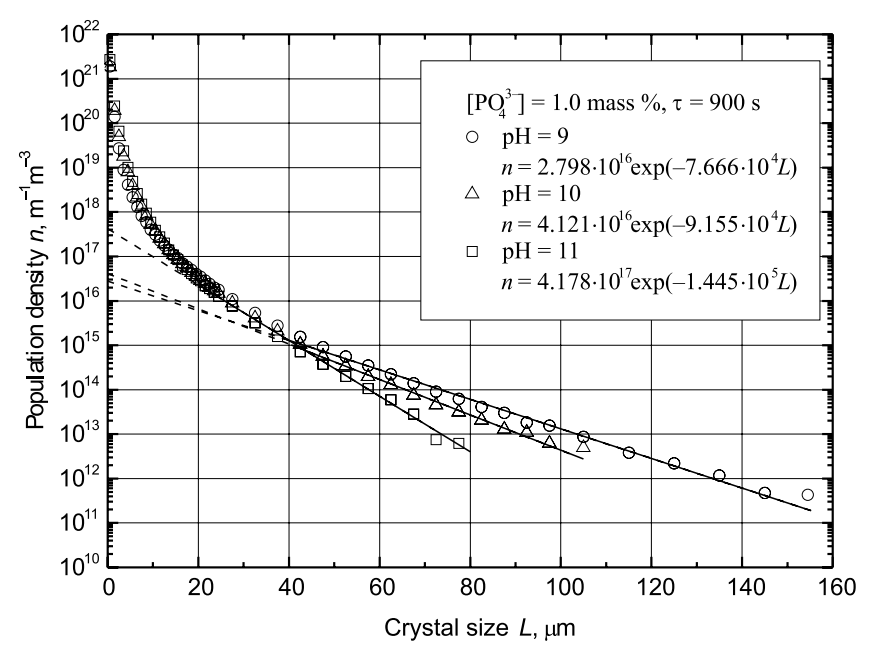

Figure 5. The effect of the process environment's $\mathrm{pH}$ on struvite crystal population density distribution in a continuous DTM MSMPR crystallizer with a jet pump driven by compressed air stream: the points - experimental data (Eq. (1)), solid lines - values calculated with Eq. (2) (see Table 2) for the crystal fractions: $L>40 \mu \mathrm{m}(\mathrm{pH} 9), L>30 \mu \mathrm{m}(\mathrm{pH} \mathrm{10)}$ and $L>20 \mu \mathrm{m}(\mathrm{pH} 11)$ 


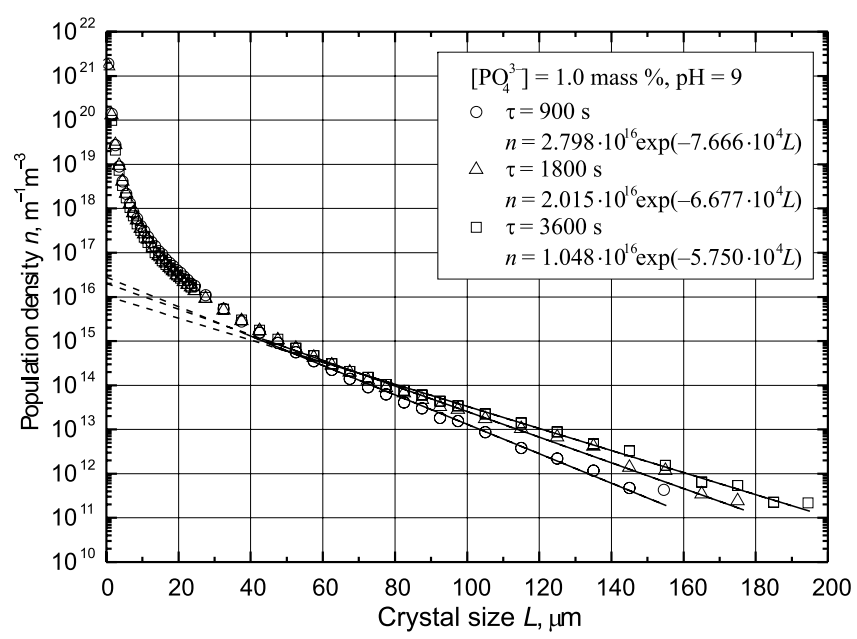

Figure 6. The effect of the mean residence time of struvite crystals in a continuous DTM MSMPR crystallizer with a jet pump driven by compressed air stream on the crystal population density distribution: the points - experimental data (Eq. (1)), solid lines values calculated with Eq. (2) (see Table 2) for the crystal fraction $L>40 \mu \mathrm{m}$

From Figs. 5 and 6 it also results that for the crystals of sizes $L<20,30$ or $40 \mu \mathrm{m}$ (depending on the appropriate environment's $\mathrm{pH}$ value) their population density distributions demonstrate significant nonlinearity of the course in $\ln n-L$ coordinate system. This characteristic, curved shape can be theoretically interpreted as the presence in a system under research of a more complex kinetics of crystal phase growth than it results from the roughly assumed, the most simplified SIG kinetic model ${ }^{2}$. A detailed kinetic analysis of this complex phenomenon will be presented in the next authors' work.

Analyzing the kinetic data presented in Table 2 one can observe a decrease of linear crystal growth rate $G$ with the increase in the process environment's $\mathrm{pH}$ and with the elongation of the mean residence time of suspension in a crystallizer. In general higher values of crystal growth rate correspond to shorter mean residence times, which agrees well with the observations concerning classical, continuous processes of mass crystallization from solutions ${ }^{\mathbf{1 - 3}}$. The average value of $G$ for t $900 \mathrm{~s}$ is $1.14 \cdot 10^{-8} \mathrm{~m} / \mathrm{s}$, while for $\tau 3600 \mathrm{~s}-$ only $3.28 \cdot 10^{-9} \mathrm{~m} / \mathrm{s}$. In turn, a relative decrease of the crystal growth rate resulting from an increase in environment's $\mathrm{pH}$ from 9 to 11 ( $\tau 900 \mathrm{~s}$ ) is $47 \%$. It is not an advantageous phenomenon. A significant decrease in the growth rate results in the manufacturing of the crystal product of clearly smaller sizes (compare the granulometric data in Table 1). A simultaneous influence of the process environment $\mathrm{pH}$ in 9-11 range and the mean residence time of suspension in a crystallizer $900<$ $\tau<3600$ s on linear growth rate of struvite crystals $G$ (in $\mathrm{m} / \mathrm{s}$ ) can be presented as an empirical correlation, Eq. (5): $G=3.34 \cdot 10^{-2} \mathrm{pH}^{-3.77} \tau^{-0.920}$

with the correlation coefficient $R^{2}=0.989$ and the mean relative error $\pm 12.7 \%$. A graphical course of this empirical dependency is presented in Fig. 7.

Nucleation rate $B$, similarly to crystal linear growth rate $G$, decreases with the elongation of the mean residence time of suspension in a crystallizer. Increase in $\tau$ value essentially decreases the working supersaturation level in a process solution. For example, elongation of $\tau$ from 900

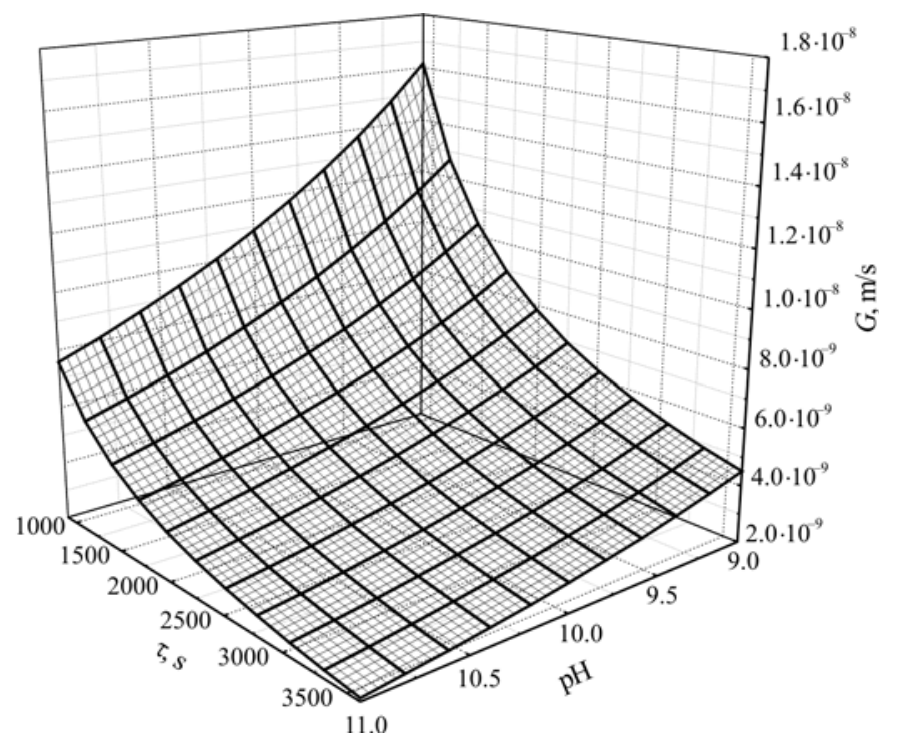

Figure 7. Simultaneous influence of $\mathrm{pH}$ and the mean residence time of suspension in a continuous DTM MSMPR crystallizer with jet pump driven by compressed air stream on linear growth rate of struvite crystals, $G$, (Eq. (4)). Concentration of phosphate(V) ions in a feed 1.0 mass $\%$, the process temperature $298 \mathrm{~K}$

to $3600 \mathrm{~s}$ caused that the nucleation rate $B$ decreased from 6 to 10 -time, depending on the current $\mathrm{pH}$ value. On the other hand, with the $\mathrm{pH}$ value increase (within the 9-11 range) the nucleation rate $B$, contrary to crystal linear growth rate $G$, increased 8 to 10 -time. Higher nucleation rate corresponds to the formation of a larger fraction of smaller crystals in a product suspension, finally resulting in a lower mean product crystal size.

For the 5-times lower concentration of phosphate(V) ions in a feed $\left(\left[\mathrm{PO}_{4}{ }^{3-}\right]=0.20\right.$ mass \%) in the same DTM MSMPR configuration nearly identical growth kinetics was observed ${ }^{19}: G=1.41 \cdot 10^{-8} \mathrm{~m} / \mathrm{s}(\mathrm{pH} 9, \tau 900 \mathrm{~s}), 9.37 \cdot 10^{-9}$ $\mathrm{m} / \mathrm{s}(\mathrm{pH} 11, \tau 900 \mathrm{~s})$ and $4.60 \cdot 10^{-9} \mathrm{~m} / \mathrm{s}(\mathrm{pH} 9, \tau 3600 \mathrm{~s})$. However, considerably lower nucleation rates were reported: $B=9.1 \cdot 10^{7}\left(1 / \mathrm{sm}^{3}\right)(\mathrm{pH} 9, \tau 900 \mathrm{~s}), 4.8 \cdot 10^{8}$ $\left(1 / \mathrm{sm}^{3}\right)(\mathrm{pH} 11, \tau 900 \mathrm{~s})$ and $1.5 \cdot 10^{7}\left(1 / \mathrm{sm}^{3}\right)(\mathrm{pH} 9$, $\tau 3600 \mathrm{~s})$. These general $G(\mathrm{pH}, \tau)$ and $B(\mathrm{pH}, \tau)$ trends were also observable in FB MSZ (Fluidized Bed with Mixed Suspension Zone) construction with a descending flow of compressed air stream in a mixing chamber ${ }^{19}$. For $\left[\mathrm{PO}_{4}{ }^{3-}\right]$ $=1.0$ mass \% these were: $G=1.33 \cdot 10^{-8} \mathrm{~m} / \mathrm{s}(\mathrm{pH} 9, \tau 900 \mathrm{~s})$, $6.62 \cdot 10^{-9} \mathrm{~m} / \mathrm{s}(\mathrm{pH} 11, \tau 900 \mathrm{~s})$ and $4.21 \cdot 10^{-9} \mathrm{~m} / \mathrm{s}(\mathrm{pH} \mathrm{9}$, $\tau 3600 \mathrm{~s}$ ), while for $\left[\mathrm{PO}_{4}{ }^{3-}\right]=0.20$ mass $\%$ the following kinetic data were reported: $G=1.34 \cdot 10^{-8} \mathrm{~m} / \mathrm{s}(\mathrm{pH} 9$, $\tau 900 \mathrm{~s}), 7.31 \cdot 10^{-9} \mathrm{~m} / \mathrm{s}(\mathrm{pH} 11, \tau 900 \mathrm{~s})$ and $3.15 \cdot 10^{-9}$ $\mathrm{m} / \mathrm{s}(\mathrm{pH} 9, \tau 3600 \mathrm{~s})$. In FB MSZ crystallizer the following nucleation rates established ${ }^{19}: B=5.7 \cdot 10^{8}\left(1 / \mathrm{sm}^{3}\right)$

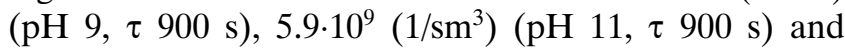
$1.4 \cdot 10^{8}\left(1 / \mathrm{sm}^{3}\right)(\mathrm{pH} 9, \tau 3600 \mathrm{~s})$ for $\left[\mathrm{PO}_{4}{ }^{3-}\right]=1.0$ mass $\%$, and $B=6.0 \cdot 10^{8}\left(1 / \mathrm{sm}^{3}\right)(\mathrm{pH} 9, \tau 900 \mathrm{~s}), 4.1 \cdot 10^{9}$ $\left(1 / \mathrm{sm}^{3}\right)(\mathrm{pH} 11, \tau 900 \mathrm{~s})$ and $3.3 \cdot 10^{7}\left(1 / \mathrm{sm}^{3}\right)(\mathrm{pH} \mathrm{9,}$ $\tau 3600 \mathrm{~s}$ ) for $\left[\mathrm{PO}_{4}^{3-}\right]=0.20$ mass $\%$.

\section{CONCLUSIONS}

The DTM MSMPR crystallizer was working stable in a continuous mode of operation. Struvite crystals of mean size $L_{\mathrm{m}}$ from ca. 14 to ca. $38 \mu \mathrm{m}$ were produced depending on the process parameter values. It was observed that 
increase in the process environment's $\mathrm{pH}$ (from 9 to 11) resulted in a disadvantageous, average 2-time decrease in the mean crystal size. On the other hand, elongation of the mean residence time of suspension in a crystallizer from 900 to $3600 \mathrm{~s}$ resulted in a significant increase in this size (even by $40 \%$ at $\mathrm{pH} 9$ ). Solid products of the relatively low size homogeneity $(\mathrm{CV})$ were removed from the crystallizer. It can be explained as an effect of complex influence of $\mathrm{pH}$ and the mean residence time of the suspension, as well as attrition and breakage within the crystal phase on the mean level of generated/discharged supersaturation in the mother solution.

The original construction of the DTM MSMPR crystallizer with a gas-liquid jet pump (lack of moving/ rotating elements), its working mode (internal circulation of suspension driven by compressed air stream) and low concentration of the solid phase in crystal magma $\left(24.6 \pm 0.4 \mathrm{~kg}\right.$ of struvite $/ \mathrm{m}^{3}$ of suspension) do not favor intensive attrition and breakage within bulk suspension. Analyzing all the individual factors determining the course of struvite reaction crystallization process in this type of crystallizer one can conclude that the main factor influencing the process run is the supersaturation level, very strongly dependent (assuming constant feed composition, constant temperature and unmodified intensity of mixing and circulating of suspension) on the process environment's $\mathrm{pH}$ and mean residence time of suspension in a crystallizer working volume.

For the identification of process kinetic parameters the simplest kinetic model elaborated for ideal MSMPR crystallizer was assumed. On this basis it was concluded that by the proper selection of $\mathrm{pH}$ and $\tau$ combinations, the linear growth rate of struvite $G$ is adjustable within the $2.06 \cdot 10^{-9}-1.45 \cdot 10^{-8} \mathrm{~m} / \mathrm{s}$ range, whereas nucleation rate $B$ within the $5.1 \cdot 10^{7}-3.2 \cdot 10^{9} 1 /\left(\mathrm{sm}^{3}\right)$ range. With the elongation of the mean residence time both kinetic parameter values decreased. With the $\mathrm{pH}$ increase nucleation rate also increased while the linear growth rate decreased.

Decrease of both kinetic parameters, $B$ and $G$, values with the elongation of mean residence time corresponded, however, to the increase in the mean size of the product crystals, $L_{\mathrm{m}}$. Lower values of linear growth rate are thus compensated with excess by longer contact time of growing crystals with the supersaturated mother solution. Simultaneously decreasing the nucleation rate values also influence the crystal growth process and self-establishing in such defined process conditions size distribution of struvite crystals advantageously. In effect more convenient conditions of mass transfer between liquid and solid phases are observed (especially convenient distribution of mass flux between the growth and nucleation processes), providing more stable conditions of crystals growth. In the process the conditions characterized by the relatively long mean residence time of the suspension in a crystallizer, a product of higher quality is manufactured. However, in such conditions the unit process yield is relatively low, thus the economic effectiveness of the production plant is lower.

Increase in environment's $\mathrm{pH}$ influences the process course and its results disadvantageously. In such conditions increase in struvite nucleation rate and decrease in crystals growth rate is observed, resulting in the increase in the number of small particles in a process system.
These small crystals demonstrate lower growth rate (compare Figs 5-6), thus altering the product size composition disadvantageously. In effect, at higher $\mathrm{pH}$ values the mean size of crystal product reaches relatively small values.

\section{ACKNOWLEDGEMENT}

The work was financed by Ministry of Science and Higher Education of Poland in 2008-2011 and 20092012 under research projects NN209 010834 and NN209 117437.

\section{NOMENCLATURE}

$B$ - nucleation rate, $1 /\left(\mathrm{m}^{3} \mathrm{~s}\right)$;

$\mathrm{CV}$ - coefficient of (crystal size) variation, defined as $100\left(L_{84}-L_{16}\right) /\left(2 L_{50}\right), \%$;

$G \quad$ - linear growth rate of crystals, $\mathrm{m} / \mathrm{s}$;

$k_{\mathrm{v}} \quad$ - volumetric shape factor of crystal;

$L \quad$ - characteristic linear size of crystal, m;

$L_{\mathrm{d}} \quad$ - dominant crystal size, $\mathrm{m}$;

$L_{\mathrm{i}} \quad$ - mean size of $i$-th crystal fraction, m;

$L_{\mathrm{m}} \quad$ - mean size of crystal population, defined as $\Sigma x_{\mathrm{i}} L_{\mathrm{i}}, \mathrm{m}$;

$L_{50}$ - median crystal size for 50 mass $\%$ undersize fraction, $\mathrm{m}$;

$\Delta L_{\mathrm{i}} \quad$ - size width of $i$-th crystal fraction, m;

$m_{\mathrm{i}} \quad$ - mass of $i$-th crystal fraction, $\mathrm{kg}$;

$M_{\mathrm{T}} \quad$ - crystal concentration in suspension (suspension density), $\mathrm{kg}_{\text {cryst }} / \mathrm{m}^{3}$;

$n_{0} \quad$ - nuclei (zero-size crystals) population density, $1 /\left(\mathrm{m} \mathrm{m}^{3}\right)$;

$n_{\mathrm{i}} \quad$ - population density of $i$-th crystal fraction, $1 /\left(\mathrm{m} \mathrm{m}^{3}\right)$;

$n(L)$ - population density (number of crystals within the specified size range in unit volume of suspension per this size range width), $1 /\left(\mathrm{m} \mathrm{m}^{3}\right)$;

$q_{\mathrm{v}} \quad$ - volumetric (out)flow rate of crystal suspension from the crystallizer, $\mathrm{m}^{3} / \mathrm{s}$;

$q_{\text {ve }}$ - volumetric (in)flow rate of a compressed air stream, $\mathrm{m}^{3} / \mathrm{s}$

$T \quad$ - process temperature, $\mathrm{K}$;

$V_{\mathrm{i}} \quad$ - volume of $i$-th crystal fraction, $\mathrm{m}^{3}$;

$V_{\mathrm{w}} \quad$ - crystallizer working volume, $\mathrm{m}^{3}$;

$x_{\mathrm{i}} \quad$ - mass fraction of crystals corresponding to mean fraction size $L_{\mathrm{i}}$.

\section{Greek letters}

$\tau \quad-$ mean residence time of suspension in a crystallizer working volume, defined as $V_{\mathrm{w}} / q_{\mathrm{v}}, \mathrm{s}$;

$\rho \quad-$ density of crystals, $\mathrm{kg} / \mathrm{m}^{3}$.
Abbreviations
CSD - Crystal Size Distribution
DTM - Draft Tube Magma (crystallizer configura- tion)
MAP -Magnesium Ammonium Phosphate (struvite)
MSMPR - Mixed Suspension Mixed Product Removal (crystallizer configuration)
SIG - Size Independent Growth (kinetic model of crystal phase growth) 


\section{LITERATURE CITED}

1. Mullin, J.W. (1993). Crystallization. Oxford: Butterworth-Heinemann.

2. Rojkowski, Z. \& Synowiec, J. (1991). Crystallization and the Crystallizers. Warszawa: WNT (in Polish).

3. Synowiec, P. (2008). Industrial Crystallization from Solution. Warszawa: WNT (in Polish).

4. Matynia, A. (1997). Crystallizers with a jet pump. Inż. Ap. Chem. 36(6), 9-14 (in Polish).

5. Koralewska, J., Piotrowski, K., Wierzbowska, B. \& Matynia, A. (2007). Nucleation and crystal growth rates of struvite in DTM type crystallizer with jet pump of descending suspension flow in a mixing chamber. Am. $J$. Agril. Biol. Sci. 2, 260-266.

6. Koralewska, J., Piotrowski, K., Wierzbowska, B. \& Matynia, A. (2007). Reaction-crystallization of struvite in a continuous liquid jet pump DTM MSMPR crystallizer with upward circulation of suspension in a mixing chamber - an SDG kinetic approach. Chem. Eng. Technol. 30, 1576-1583. DOI: 10.1002/ceat.200700229.

7. Koralewska, J., Piotrowski, K., Wierzbowska, B. \& Matynia, A. (2009). Kinetics of reaction-crystallization of struvite in the continuous Draft Tube Magma type crystallizers - influence of different internal hydrodynamics. Chinese J. Chem. Eng. 17, 330-339. DOI: 10.1016/ S1004-9541(08)60212-8.

8. Matynia, A., Piotrowski, K., Ciesielski, T. \& Liszka, R. (2009). New constructions of crystallizer with a compressed air driven jet-pump in the phosphorus recycling technology. Przem. Chem. 88, 505-508 (in Polish).

9. Randolph, A.D. \& Larson, M.A. (1988). Theory of Particulate Processes: Analysis and Techniques of Continuous Crystallization. New York, USA: Academic Press.

10. Doyle, J. \& Parsons, S.A. (2002). Struvite formation, control and recovery. Wat. Res. 36, 3925-3940. DOI: 10.1016/S0043-1354(02)00126-4.

11. Parsons, S.A. (2001). Recent scientific and technical developments: struvite precipitation. CEEP Scope Newslett. 41, 15-22.

12. Matynia, A., Koralewska, J., Piotrowski, K. $\&$ Wierzbowska, B. (2006). The influence of the process parameters on the struvite continuous crystallization kinetics., Chem. Eng. Comm. 193, 2, 160-176. DOI: 10.1080/009864490949008.

13. Le Corre, K.S., Valsami-Jones, E., Hobbs, P. \& Parsons, S.A. (2009). Phosphorus recovery from wastewater by struvite crystallization: A review. Crit. Rev. Environ. Sci. Technol. 39, 433-477. DOI: 10.1080/ 10643380701640573.

14. Grzmil, B. \& Wronkowski, J. (2004). Process for removing and recovering phosphates from wastewaters. Przem. Chem. 83, 275-280 (in Polish).

15. Gorazda, K., Wzorek, Z., Jodko, M. \& Nowak, A.K. (2004). Struvite - manufacturing methods. Chemik 57, 317-320 (in Polish).

16. de-Bashan, L.E. \& Bashan, Y. (2004). Recent advances in removing phosphorus from wastewater and its future use as fertilizer. Wat. Res. 38, 4222-4246. DOI: 10.1016/j.watres.2004.07.014.

17. Matynia, A., Piotrowski, K., Liszka, R. \& Ciesielski, T. (2009). Reaction crystallization of struvite in a continuous DTM type crystallizer with jet-pump driven by com- pressed air. In Proceedings of $36^{\text {th }}$ International Conference of Slovak Society of Chemical Engineering, Tatranské Matliare, Slovakia, CD-ROM No. 067, 067.1-067.10.

18. Matynia, A., Liszka, R., Ciesielski, T. \& Piotrowski, K. (2009). Reaction crystallization of struvite from diluted water solutions in a continuous DTM-type crystallizer with jet pump driven by compressed air. Inż. Ap. Chem. 48(4), 83-84.

19. Matynia, A., Mazieńczuk, A., Wierzbowska, B., Kozik, A. \& Piotrowski, K. (2010). New crystallizers with a compressed air-driven jet pump in the process of precipitating struvite from diluted water solutions containing phosphate ions. Chemik 64, 753-758. 